\title{
Stevens Johnson Syndrome Like Skin Lesions in a Patient With Systemic Lupus Erythematosus After Hydroxychloroquine Treatment, a Case
}

\author{
Orhan Zengin ${ }^{1}$, Mustafa Erkut Önder ${ }^{2}$, Abdi İbrahim Halil Sönmez ${ }^{3}$, İbrahim \\ Halil Türkbeyler ${ }^{4}$ \\ ${ }^{1}$ Dr Ersin Aslan Research Hospital, Department of Rheumatology, Gaziantep, Turkey. \\ ${ }^{2}$ Manisa City Hospital, Department of Rheumatology, Manisa, Turkey. \\ ${ }^{3}$ Abdi İbrahim Halil Sönmez, Gaziantep University, Department of Hematology, Gaziantep, \\ Turkey. \\ ${ }^{4}$ Dr Ersin Aslan Research Hospital, Department of Geriatrics, Gaziantep, Turkey.
}

\begin{abstract}
Stevens Johnson Syndrome (SJS) is a clinical syndrome with a high morbidity and mortality, characterized by exfoliation of the skin, generally due to medications, and less frequently due to infections. Most frequently sulphonamides, penicillin, and anti-convulsants were among blamed medications. Systemic lupus erythematosus (SLE) is a systemic auto-immune disorder and skin involvement is frequently seen. Hydroxychloroquine (HCQ) is an important agent which is used in the treatment of SLE and which increases survival. Its side effects include skin reactions. We present here a case of SJS like skin lesions in a 38-year-old female patient with SLE after receiving $H C Q$.
\end{abstract}

Key words: Stevens Johnson Syndrome, Systemic lupus erythematosus, Hydroxychloroquine,

Skin lesion.

\section{Introduction}

Stevens Johnson syndrome (SJS) is severe cutaneous adverse reaction, mainly caused by drugs but also related to infections and unidentified causes. It is characterized by an acute onset of erythema with detachment of the epidermis and epithelia of mucous membranes resulting in extensive areas of denuded skin (1). Stevens Johnson Syndrome and toxic epidermal necrolysis (TEN) are considered to be severity variants of the same disease entity with SJS being the milder and TEN the most severe form (2). The disease has been related to some drugs, such as the sulphonamides, penicillin, salicylates, antiretroviral drugs and anti-convulsants, and there is an association with herpes simplex and mycoplasma pneumoniae infections (3). Systemic lupus erythematosus (SLE) is an autoimmune disease characterized by the presence of autoantibodies that can cause tissue damage in multiple organs. The skin is the second most commonly affected organ involved in SLE, including malar rash, subacute cutaneous lupus, discoid lupus, bullous lesions, periungual erythema (4).

\footnotetext{
*Corresponding author: Orhan Zengin, Tel: +90 34236065 65, e-mail: drorhanzengin@ gmail.com, ORCID ID: 0000-0002-6207-8749.
} 
Hydroxychloroquine (HCQ) is an antimalarial drug with immunosuppressive and anti-inflammatory effects that is used in the treatment of SLE, rheumatoid arthritis and other disorders of connective tissues. It has protective effects against disease progression in SLE. Side effects include gastrointestinal disturbances, headache, retinopathy and skin rash (5). There are very few cases of SJS due to HCQ in the medical literature. We present here a case of a patient with SLE in whom serious and life-threatening skin reactions like Stevens Johnson Syndrome after receiving HCQ.

\section{Case report}

A 38-year-old female patient was admitted with complaints of fatigue, swelling and pain in the wrists and metacarpophalangeal (MCP) joints, and redness at the face and arms which became more prominent after exposure to sunlight. She did not have any co-existent diseases, but her mother had SLE. The patient had swelling, sensitivity and limitation of motion of the right wrist, left 1st MCP, right 1st, 2nd, and 3rd MCP, and a malar rash and no other findings were detected in her physical examination. Laboratory values were as follows; hemoglobin $8.2 \mathrm{~g} / \mathrm{dl}$ (12.5-13.5), leukocytes $\quad 4.500 / \mathrm{mm}^{3} \quad$ (4500-10000), platelets $98.000 / \mathrm{mm}^{3} \quad(140000-440000)$, urea: $11 \mathrm{mg} / \mathrm{dl}$ (10-50), creatinine 0.4 $\mathrm{mg} / \mathrm{dl}$ (0.4-1.1), aspartate aminotransferase (AST) $49 \quad$ IU/1 (4-44), alanin aminotransferase (ALT) 56 IU/1 (4-44), total protein $6.1 \mathrm{~g} / \mathrm{dl}$ (6-8.2), albumin 3.3 $\mathrm{g} / \mathrm{dl}$ (3.5-5.1). C-reactive protein was 15 $\mathrm{mg} / \mathrm{dl}$ (0-5), erythrocyte sedimentation rate (ESR) was $34 \mathrm{~mm} / \mathrm{h}$ (1-20), Rheumatoid factor (RF) $9 \mathrm{IU} / \mathrm{ml}$ (0-15). Autoantibodies were planned to be measured, and treatment with $5 \mathrm{mg}$ /day prednisolon was started. The joint complaints receded and the patient was admitted again 2 weeks later. Anti citrullinated cyclic peptide (Anti-CCP) $5 \mathrm{IU} / \mathrm{ml}$ (0-15), antinuclear antibody (ANA) homogenously positive 1:1280 titres $(>1 / 80)$, anti-dsDNA 5 (020) U/ml, complement 3 (c3) $0.723 \mathrm{~g} / \mathrm{l}$ (0.9-1.8) and complement 4 (C4) was $0.249 \mathrm{~g} / 1$ (0.1-0.4). Direct and indirect Coombs tests were positive. Normochrome normocytic erythrocytes were seen at the peripheral blood smear, with 8-9 platelets at each field. Urinalysis was normal. Anti SS-A, Anti-histone, Anti-centromer, Antijo1, Anti-SM, Anti-SCl-70, Anti-SS-B were negative. A diagnosis of SLE was decided with arthritis of the wrist and small joints of the hand, malar rash, photosensitivity, anemia, thrombocytopenia, positive ANA, low c3, positive Coombs. Hydroxychloroquine $400 \mathrm{mg} /$ day was added to the treatment. The patient was admitted again 7 days later with fever and rash. Her temperature was $39.8^{\circ} \mathrm{C}$ with maculopapular and bullous lesions all over her body, which became denser on the neck, knees, chest and back, and mucositis on the lips and inside the mouth. Her blood pressure was $100 / 80 \mathrm{mmHg}$, pulse $70 / \mathrm{min}$, and rate of breathing that was $14 / \mathrm{min}$. Findings from further physical examinations were unremarkable. Laboratory examination results were not different from earlier values.

Hydroxychloroquine treatment was terminated and methyl prednisolon $1 \mathrm{~g} / \mathrm{day}$ IV was administered for 3 days, which was maintained at a dose of $60 \mathrm{mg} / \mathrm{day}$. The skin lesions disappeared in time, and the patient was discharged cured and without fever. 


\section{Discussion}

Stevens Johnson Syndrome is a clinical picture with very high morbidity and mortality, characterized by exfoliation of the skin, generally due to medications, and less frequently to infections (3). It runs a course of epidermal exfoliation, conjunctivitis, mucosal membrane involvement and fever. While SJS is a milder disorder where less than $10 \%$ of the body surface is involved with mucous membrane erosions and vesicular lesions, TEN is a clinical entity where more than $30 \%$ of the total body surface is involved with lesions resembling superficial burns due to coalescence of erosions and vesicles. An involvement of body surface between $10-30 \%$ is considered as an overlap of SJS - TEN. The etiology is frequently associated with drug use. These medication include sulphonamides and penicillin group among antibiotics, phenytoin, carbamazepine and barbiturates among anticonvulsants, non-steroidal antiinflammatory drugs, allopurinol and antifungals. Other causes include mycoplasma, herpes, varicella and measles infections, neoplasies, autoimmune diseases such as SLE, and radiotherapy (6). Our patient exfoliative skin lesions, fever and oral mucosal involvement is considered SJS. There are no universally accepted diagnostic criteria for Stevens Johnson syndrome/toxic epidermal necrolysis (SJS/TEN). However, the diagnosis of SJS/TEN would be appropriate in a patient with the following clinical features (7);

1) History of drug exposure or febrile illness,

2) A prodrome of acute-onset febrile illness and malaise.

3) A painful rash that progresses rapidly.
4) Erythematous macules, targetoid lesions, or diffuse erythema progressing to vesicles and bullae.

5) Positive Nikolsky sign and/or "bulla spread sign."

6) Oral, ocular, and/or genital mucositis with painful mucosal erosions.

7) Necrosis and sloughing of the epidermis of varying degree.

Systemic lupus eryhtematosus is a prototype of autoimmune diseases with a multisystem involvement. The clinical spectrum is quite variable, from skin involvement to joint involvement, and from organ involvement to a lifethreatening clinical picture. The second most frequently seen organ involvement is the skin. Acute cutaneous lupus erythematosus (ACLE) can be categorized into localized ACLE, generalized ACLE and toxic epidermal necrolysis-like ACLE (8).

Hydroxychloroquine is a reliable drug which was proven to be effective in the treatment of SLE. It is an anti-malarial drug reported to have anti-inflammatory and immunosuppressive effects. The mechanisms of action of HCQ are, Tolllike receptor activation antagonism, inhibition of interferon-alpha expression, IFN-alpha mediated pathways inhibition (9). The most important toxic effect is retinal damage. For this reason, periodic ophthalmologic examinations should be done every 4-6 months during treatment. Other important side effects include fulminant hepatic failure, autotoxicity, neuromyotoxicity and skin reactions (5).

In the literature the association of SLE and SJS has numerous cases reported. Some patients with SLE present with SJS. Other SLE patients, depending on the drug they developed SJS $(10,11)$. Few cases have 
been reported SJS associated with HCQ. The data of the German Hospital between 1990 and 2006 was evaluated in this study by Ziemer et al, and a history of SLE was detected in 17 of 1366 patients. The number of female patients was larger, with a mean age of 49.2 and increased positivity of anti-La and Anti-Ro. Also, 13 of the 17 patients were taking immunosuppressive medications, with a history of drug intake in 15 (88\%). Stevens Johnson Syndrome was more frequently seen in young female patients and in those with positive Anti-Ro and Anti-La in other reported cases in the literature. Stevens Johnson Syndrome had developed in a 31year-old patient who was being followedup due to SLE, with two probable causing medications: co-trimoxazole and HCQ. On the other hand, HCQ is the only etiologic agent in another patient. This patient was a 50-years-old female followed-up for CLE with positive ANA and Anti-Ro. She had had skin lesions resembling erythema multiforme before the incident, after which SJS had developed with generalized mucocutaneous involvement (10). Lateef have been reported a 67 -year-old woman with SLE after treatment of HCQ developed acute generalized pustulosis exanthematous and TEN overlap. And the patient successfully treated with steroid and intravenous immunoglobulin (IVIG) therapy (12). Simsek reported a 28-yearold woman patient with SLE after the treatment of prednisolone and HCQ developed TEN like reaction (13). Lastly in Leckie's study, a 65 years old diagnosed with rheumatoid athritis for almost 9 year, after 2 weeks addition of $200 \mathrm{mg} /$ day of HCQ and developing SJS, it reported that she respond with steroids (14). In addition, in previous studies, there are cases shows the association of using steroid to develop
SJS in SLE patients $(13,15,16,17,18)$.

Our patient had HCQ and prednisolone intake. There is a decline and disappearing of clinical complaints after prednisolone treatment, the formation of skin lesions was thought to be developed after the treatment of HCQ.

The mechanisms leading to SJS are not yet fully understood, but a modification of the structure of the drug could thus alter this presentation due to poor recognition of the drug molecule by major histocompatibility complex (MH C) or the T cell receptor (19). This recognition by the lymphocyte receptors leads to their activation (20). The other important mechanism is an increase in keratinocyte cell death. This is caused by an increased FasL and Fas expression in the keratinocytes (21). Also, immunochemical examination of skin biopsies have shown an increased expression of FasL in all patients with SLE in comparison with controls (22). A triggering agent such as a medication may increase apoptotic ligand production of keratinocytes and may cause apoptosis with Fas-FasL binding (23).

There is still no specific therapy. A satisfactory benefit from systemic steroids and immunosuppressive treatment was not reported. The recent introduction of IVIG therapy in these cases is a safe alternative treatment for these patients (24). The antibodies in IVIG were shown to bind to Fas, thus inhibiting FasL binding to Fas. Also, IVIG was found to strongly inhibit apoptosis by inhibiting in vitro Fas-FasL binding $(22,25)$. Our patient could be successfully treated with steroids and supportive therapy. In conclusion, SJS may occur frequently in patients with SLE. The etiology of these patients HCQ and steroid therapy should be noted that may 
take place.

\section{Conflict of interest}

The authors declare that no conflict of interest exists.

\section{Acknowledgement}

No institution has given financial support to the study. All researchers contributed equally to the study.

\section{References}

1. Bastuji-Garin S, Rzany B, Stern RS et al. Clinical classi cation of cases of toxic epidermal necrolysis, Stevens-Johnson syndrome, and erythema multiforme. Arch Dermatol 1993; 129:92-6.

2. Mockenhaupt M. The current understanding of Stevens-Johnson syndrome and toxic epidermal necrolysis. Expert Rev Clin Immunol 2011; 7:80315.

3. Tay YK, Huff JC,Weston WL. Mycoplasma pneumoniae infection is associated with stevensjohnson sendrome, not erythema multiforme (von hebra). J am Acad dermatol 1996;35:757-60.

4. Andrea Fava, Michelle Petri. Systemic Lupus Erythematosus: Diagnosis and Clinical Management. J Autoimmun. 2019;96:1-13.

5. John B. Imboden, David B. Hellmann, John H.Stone. Current diagnozis and treatment rheumatology 3. Edition. 2013 200-201.

6. Chan HL, Stern RS, Arndt KA, et al. The incidence of erythema multiforme, StevensJohnson syndrome, and toxic epidermal necrolysis. A population-based study with particular reference to reactions caused by drugs among outpatients. Arch Dermatol 1990;126:43-47.

7. Schwartz RA, McDonough PH, Lee BW. Toxic epidermal necrolysis: Part II. Prognosis, sequelae, diagnosis, differential diagnosis, prevention, and treatment. J Am Acad Dermatol 2013; 69:187.1.

8. Gilliam JN, Sontheimer RD. Distinctive cutaneous subsets in the spectrum of lupus erythematosus. J Am Acad Dermatol. 1981;4:4715.

9. Akhavan PS, Su J, Lou W, et al. The early protective effect of Hydroxychloroquine on the risk of cumulative damage in patients with systemic lupus erythematosus. J Rheumatol. 2013 Jun;40(6):831-41.
10. M.ziemer
S.H.Kardaun, Y.liss,

M.mockenhaupt. Stevens-Johnson syndrome and toxic epidermal necrolysis in patients with lupus erythematosus: a descriptive study of 17 cases from a national registry and review of the literature. British journal of dermatology British Association of Dermatologists 2012 166, pp575-600.

11. Lee HY, Tey HL, Pang SM, Thirumoorthy T. Systemic lupus erythematosus presenting as Stevens-Johnson syndrome and toxic epidermal necrolysis: a report of three cases. Lupus. 2011 May;20(6):647-52.

12. Lateef A, Tan KB, Lau TC. Acute generalized exanthematous pustulosis and toxic epidermal necrolysis induced by hydroxychloroquine. Clinical Rheumatology. 2009 Dec; 28(12):1449-52.

13. Simsek I, Cinar M, Erdem H, et al. Efficacy of plasmapheresis in the treatment of refractory toxic epidermal necrolysis like acute cutaneous lupus erythematosus. Lupus. 2008; 17:605Y606.

14. Leckie MJ, Rees RG. Stevens-Johnson syndrome in association with hydroxychloroquine treatment for rheumatoid arthritis. Rheumatology (Oxford) 2002 Apr; 41(4):473-4.

15. Samimi SS, Siegfried E. StevensJohnson syndrome developing in a girl with systemic lupus erythematosus on high-dose corticosteroid therapy. Pediatr Dermatol. 2002 JanFeb;19(1):52-5.

16. Lee HY, Tey HL, Pang SM, et al. Systemic lupus erythematosus presenting as Stevens-Johnson syndrome and toxic epidermal necrolysis: a report of three cases. Lupus. 2011; 20:647Y652.

17. Mandelcorn R, Shear NH. Lupus-associated toxic epidermal necrolysis: a novel manifestation of lupus? J Am Acad Dermatol. 2003; 48:525Y529.

18. Ryan E, Marshman G, Astill D. Toxic epidermal necrolysis-like subacute cutaneous lupus erythematosus. Australas J Dermatol. 2012; 53:303 Y306.

19. Wei CY, Chung WH, Huang HW, Chen YT, Hung SI. Direct interaction between HLA-B and carbamazepine activates $\mathrm{T}$ cells in patients with Stevens-Johnson syndrome. J Allergy Clin Immunol 2012; 129(6): 1562-9 e5.

20. Wu Y, Sanderson JP, Farrell J, et al. Activation of $\mathrm{T}$ cells by carbamazepine and carbamazepine metabolites. J Allergy Clin Immunol 2006; 118(1): 233-41.

21. Rutter A, Luger TA. High-dose intravenous immunoglobulins: an approach to treat severe immune-mediated and autoimmune disease. J Am Acad Dermatol 2001; 44:1010-1024.

22. Viard I, Wehrli P. Bullani R, et al. Inhibition of toxic epidermal necrolysis by blockade of CD95 with human intravenous immunoglobulin. Science 1998; 282:490-493.

23. Metry DW, Jung P, Levy ML. Use of intravenous immunoglobulin in children with Stevens-Johnson syndrome and toxic epidermal 
O. Zengin et al.

necrolysis: seven cases and review of the literatüre. Pediatrics 2003; 112(6 Pt 1):1430-1436.

24. Kazatchkine MD, Kaveri SV. Immunomodulation of autoimmune and inflammatory diseases with intravenous immune globulin. N Engl J Med 2001; 345:747-755.

25. Tanaka M, Suda T, Haze K, et al. Fas ligand in human serum. Nat Med 1996; 2:317-322. 